\title{
SNAREs - engines for membrane fusion
}

\section{Reinhard Jahn* and Richard H. Scheller*}

Abstract | Since the discovery of SNARE proteins in the late 1980s, SNAREs have been recognized as key components of protein complexes that drive membrane fusion. Despite considerable sequence divergence among SNARE proteins, their mechanism seems to be conserved and is adaptable for fusion reactions as diverse as those involved in cell growth, membrane repair, cytokinesis and synaptic transmission. A fascinating picture of these robust nanomachines is emerging.

$\mathrm{AAA}+$ proteins

('ATPases associated with various cellular activities' proteins). A superfamily of proteins with one or two nucleotide-binding domains, which often form ring-like oligomers and function as chaperones in diverse cellular processes. They can unfold aggregates or tightly packed structures.

\section{Palmitoylation}

A post-translational modification of proteins in which a palmitate fatty acyl chain is covalently attached to a cysteine side chain by a thioester bond

\footnotetext{
*Department of Neurobiology, Max Planck Institute for Biophysical Chemistry, Am Fassberg. 37077 Göttingen, Germany. ${ }^{*}$ Genentech Inc., South San Francisco, California 94080 , USA.

Correspondence to R.J. e-mail: rjahn@gwdg.de doi:10.1038/nrm2002 Published online 16 August 2006
}

Eukaryotic cells contain membrane-enclosed organelles that communicate with each other through the exchange of trafficking vesicles. Trafficking usually involves the generation of a vesicle from a precursor membrane, the transport of the vesicle to its destination and, last, the fusion of the vesicle with the target compartment. Despite an enormous diversity in the size and shape of the organelles, the basic reactions - budding and fusion - are carried out by multiprotein complexes that consist of protein families that have been conserved throughout eukaryotic evolution ${ }^{1}$.

Since the late 1980s, when SNARE (soluble $\mathrm{N}$-ethylmaleimide-sensitive factor attachment protein receptor) proteins were first characterized, rapid progress has identified SNAREs as key elements in membrane fusion. Although extracellular membrane fusion and the fusion events of mitochondria and peroxisomes involve unrelated proteins, SNAREs seem to mediate membrane fusion in all of the trafficking steps of the secretory pathway. A mechanistic molecular model of SNARE-mediated membrane fusion has emerged that - although not undisputed - is supported by a steadily increasing body of evidence (see the TIMELINE for the key milestones in the field). According to this model, SNARE proteins that are localized in opposing membranes drive membrane fusion by using the free energy that is released during the formation of a fourhelix bundle. The formation of this bundle leads to a tight connection of the membranes that are destined to fuse, and initiates the membrane merger. The recycling of SNAREs is achieved through the dissociation of the helical bundle, which is mediated by the AAA+ protein NSF ( $N$-ethylmaleimide-sensitive factor).

In this article, we give an overview of the structure of SNARE proteins, and then describe how the emerging biophysical features of the SNARE cycle are providing an increasingly coherent picture of SNARE-mediated membrane fusion. For further information, the reader is referred to recent reviews ${ }^{2-4}$ that cover some of the topics that are addressed here in more depth.

\section{SNARE structure}

SNARE proteins form a superfamily of small proteins with 25 members in Saccharomyces cerevisiae, 36 members in humans and 54 members in Arabidopsis thaliana. They have a simple domain structure, and a characteristic of SNAREs is the SNARE motif - an evolutionarily conserved stretch of 60-70 amino acids that are arranged in heptad repeats. At their C-terminal ends, most SNAREs have a single transmembrane domain that is connected to the SNARE motif by a short linker. Many SNAREs have independently folded domains that are positioned N-terminal to the SNARE motif and that vary between the subgroups of SNAREs (FIG. 1; reviewed in REFS 2,3).

Although this prototypic structure applies to most SNAREs, there are important exceptions. A subset of SNAREs (including the evolutionarily younger 'brevins'5) lacks the N-terminal domain. Another subset lacks transmembrane domains, but most of these SNAREs contain hydrophobic post-translational modifications that mediate membrane anchorage. These SNAREs include a small group that is represented by the neuronal SNARE SNAP-25 (25-kDa synaptosomeassociated protein), which contains two different SNARE motifs that are joined by a flexible linker that is palmitoylated. In the S. cerevisiae SNARE Ykt6, the transmembrane domain is replaced by a CAAX box that is farnesylated ${ }^{6}$. Intriguingly, SNAREs that carry transmembrane domains can also be palmitoylated, which has recently been shown to protect SNAREs from ubiquitylation and subsequent degradation ${ }^{7}$. 
Timeline | The discovery of SNAREs and the role of SNARE cycling in membrane fusion

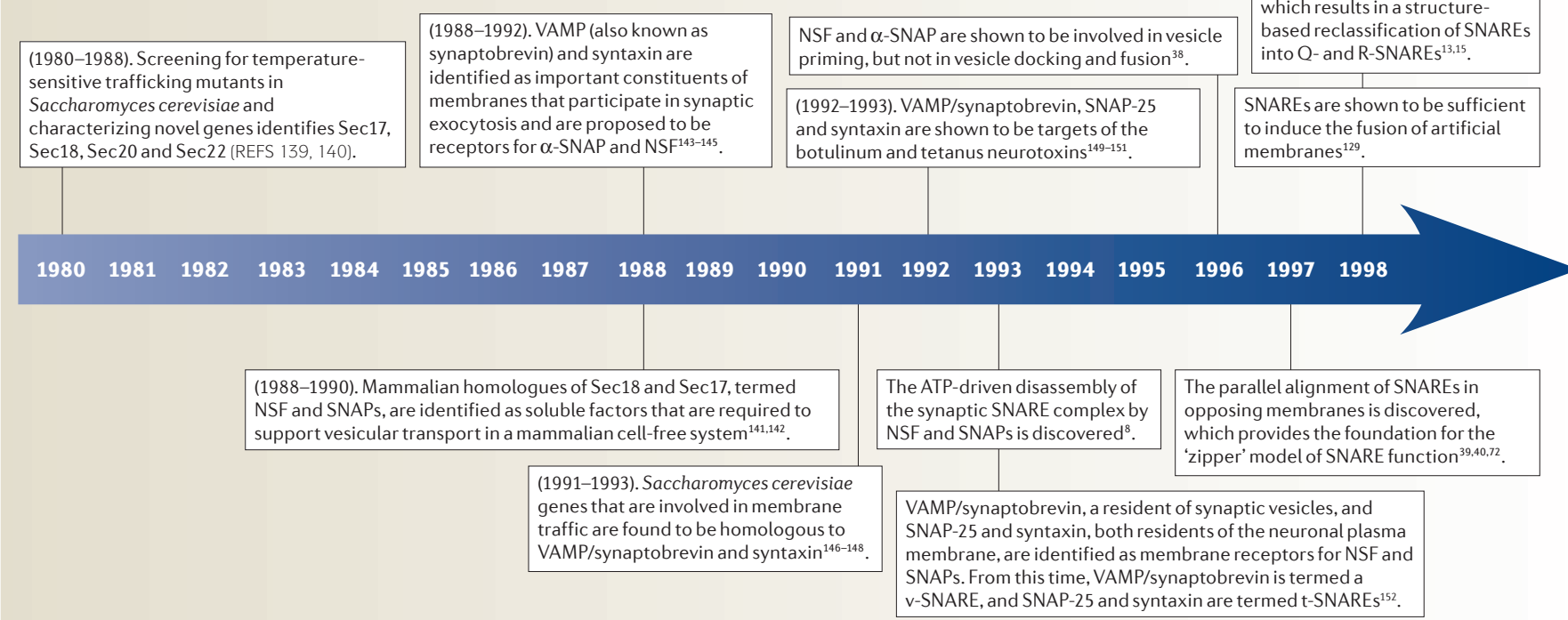

NSF, N-ethylmaleimide-sensitive factor; SNAP, soluble NSF attachment protein; SNAP-25, 25-kDa synaptosome-associated protein; SNARE, soluble NSF attachment protein receptor; $\mathrm{t}$, target membrane; v, vesicle membrane; VAMP, vesicle-associated membrane protein.

CAAX box

A C-terminal motif of four amino acids - cysteine (C),

two aliphatic amino acids (AA) and then any amino acid $(X)-$

that is recognized as a

substrate by

farnesyltransferase and

geranylgeranyltransferase I.

Farnesylation

A post-translational

modification of proteins in which a 15-carbon farnesyl

residue is covalently attached to the cysteine of a CAAX-box motif by a thioester bond.
SNARE motifs. Key to understanding the function of SNAREs in membrane fusion was the discovery that different sets of SNAREs that are present in two opposing membranes associate into complexes that are subsequently disassembled by NSF. Originally, it was assumed that there was a strict separation between SNAREs on the 'donor' compartment and the 'acceptor' compartment, which led to their functional classification as V-SNAREs (vesiclemembrane SNAREs) or t-SNAREs (target-membrane SNAREs $)^{8}$. However, this terminology is not useful in describing homotypic fusion events, and certain SNAREs function in several transport steps with varying partners. For example, the S. cerevisiae SNARE Sec22 functions in both anterograde and retrograde traffic between the endoplasmic reticulum (ER) and the Golgi apparatus. In anterograde traffic, Sec22 is colocalized with Bos 1 and Bet1 on the transport vesicle, but only Bet1 was classified as a v-SNARE, whereas Bos1 and Sec22 were classified as t-SNAREs ${ }^{9}$. In retrograde transport, Sec22 is thought to be the sole functional SNARE on the transport vesicle ${ }^{10-12}$. A more rigorous and invariant classification comes from understanding SNARE complexes.

Complex formation is mediated by the SNARE motifs, and is associated with conformational and free-energy changes. When SNAREs are monomeric, SNARE motifs are unstructured. However, when appropriate sets of SNAREs are combined, the SNARE motifs spontaneously associate to form helical core complexes of extraordinary stability (reviewed in REF. 3). The crystal structures of two, only distantly related, SNARE core complexes have revealed a remarkable degree of conservation ${ }^{13,14}$ (FIG. 2). Core complexes are represented by elongated coiled coils of four intertwined, parallel $\alpha$-helices, with each helix being provided by a different SNARE motif. The centre of the bundle contains 16 stacked layers of interacting side chains (FIG. 2). These layers are largely hydrophobic, except for a central ' 0 ' layer that contains three highly conserved glutamine $(\mathrm{Q})$ residues and one highly conserved arginine (R) residue. Accordingly, the contributing SNARE motifs are classified into Qa-, Qb-, Qc- and R-SNAREs ${ }^{15,16}$ (FIG. 1a). Functional SNARE complexes that drive membrane fusion are hetero-oligomeric, parallel four-helix bundles, and each bundle is invariant, requiring one of each of the Qa-, Qb-, Qc- and R-SNAREs. Indeed, a phylogenetic analysis of SNARE sequences from $S$. cerevisiae, A. thaliana and mammals showed that these four SNARE subfamilies are highly conserved and diverged early in eukaryotic evolution ${ }^{2,16}$.

Due to their amphiphilic nature, SNARE motifs can also associate in other combinations that result in helical bundles that are less stable than core complexes. Particularly noteworthy are the complexes that are formed by the neuronal SNAREs. These include a Qaaaa complex (an antiparallel four-helix bundle ${ }^{17}$ ), a Qabab complex (a parallel four-helix bundle ${ }^{18}$ ), a Qaabc complex (a parallel four-helix bundle with some disordered regions $s^{19,20}$ ) and, surprisingly, an antiparallel QabcR complex ${ }^{21,22}$. These complexes might not have the correct membrane topology or they might not contribute sufficient energy to drive membrane fusion. They therefore probably represent 'off-pathway' reactions (see later; see also REF. 3).

$N$-terminal domains. Unlike the conserved SNARE motifs, there are different types of independently folded N-terminal domain ${ }^{23,24}$. Qa-SNAREs, and some Qb- and Qc-SNAREs, have N-terminal antiparallel three-helix bundles (FIG. 1a,b). These bundles can vary in length and are connected to the SNARE motif by a flexible linker. By contrast, the $\mathrm{N}$-terminal domains of many R-SNAREs have profilin-like folds ${ }^{23-26}$ (FIG. 1C), which are sometimes referred to as longin domains and are also found 
a

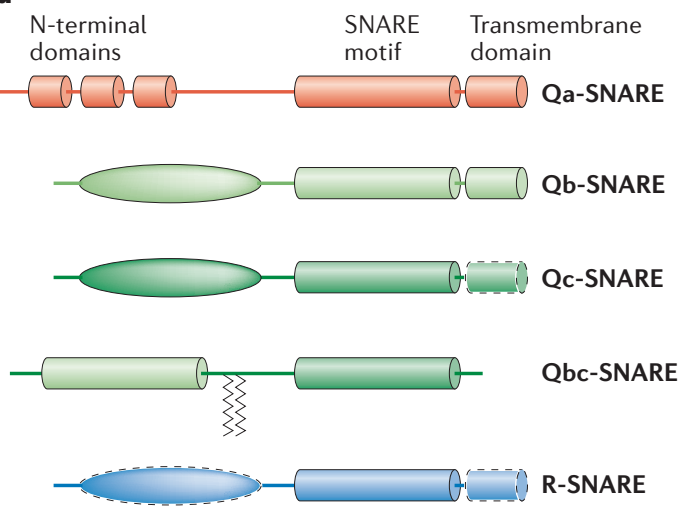

b

$\mathrm{N}$-terminal domain of syntaxin-1

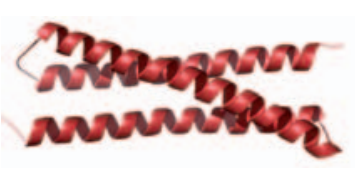

'Closed' conformation of syntaxin-1

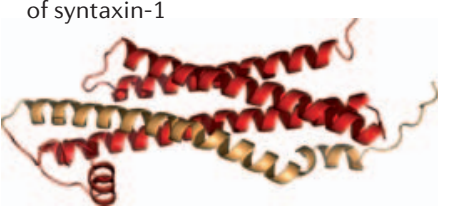

C

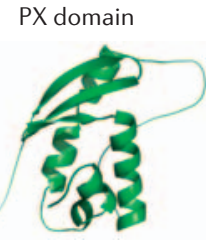

Longin domain

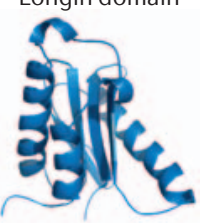

Figure 1 | The structures of SNAREs. a | The domain structure of the SNARE (soluble N-ethylmaleimide-sensitive factor attachment protein receptor) subfamilies. Dashed domain borders highlight domains that are missing in some subfamily members. Qa-SNAREs have N-terminal antiparallel three-helix bundles. The various N-terminal domains of Qb-, Qc-and R-SNAREs are represented by a basic oval shape. Qbc-SNAREs represent a small subfamily of SNAREs — the SNAP-25 (25-kDa synaptosome-associated protein) subfamily — that contain one Qb-SNARE motif and one Qc-SNARE motif. These motifs are connected by a linker that is frequently palmitoylated (zig-zag lines in the figure), and most of the members of this subfamily function in constitutive or regulated exocytosis. In this, and the following figures, the same colour scheme is used for the SNARE subfamilies (Qa-SNARE, red; Qb-SNARE, light green; Qc-SNARE, dark green; and R-SNARE, blue). b|The upper panel shows the three-dimensional structure of the isolated $\mathrm{N}$-terminal domain of syntaxin-1 (REF. 137). This structure is an N-terminal three-helix bundle that is typical of Qa-SNAREs, as well as of some Qb-and Qc-SNAREs. The lower panel shows the 'closed' conformation of syntaxin-1, in which the N-terminal domain of syntaxin-1 (red, as in the upper panel) is associated with part of its own SNARE motif (beige structure; absent in the upper panel). This structure was solved as part of the structure of the Munc18-syntaxin-1 complex ${ }^{138} . \mathbf{c}$ |Three-dimensional structures of the N-terminal domains of other SNAREs, which exemplify the structural diversity that exists. The upper panel shows the Phox-homology (PX) domain of the Qc-SNARE Vam7 (REF. 27), which seems to be unique for this particular SNARE. The lower panel shows the profilin or longin domain of the R-SNARE Ykt6 (REF. 26). The authors are indebted to F. Gräter (Max Planck Institute for Biophysical Chemistry, Göttingen, Germany) for help in preparing the figure.

in proteins that are unrelated to SNAREs ${ }^{5}$. R-SNAREs that have longin domains are conserved among all eukaryotes, whereas the evolutionarily younger brevin R-SNAREs lack a folded N-terminal domain and have only a few amino acids beyond their SNARE motif 5 . The S. cerevisiae Qc-SNARE Vam7 is unique in that it has a Phox-homology (PX) domain ${ }^{27}$ that is responsible for membrane binding (FIG. 1c). The presence of further folds in the N-terminal domains of Qb- and Qc-SNAREs cannot be excluded, because some of these domains are divergent in sequence and length.

What is the function of the $\mathrm{N}$-terminal domains? Some N-terminal domains of the three-helix-bundle type reversibly associate with the SNARE motif of the same SNARE to form a 'closed' conformation, which prevents the SNARE motif from forming a SNARE complex $^{28,29}$ (FIG. 1b). However, others cannot assume closed conformations (reviewed in REFS 5,24), which indicates that this conformation is not essential for SNARE function. The N-terminal domains might function as recruitment platforms for the binding of other proteins such as SM (Sec1/Munc18-related) proteins. SM proteins are a small family of soluble proteins that have a conserved structure and are essential for fusion ${ }^{30}$. Surprisingly, the crystal structures of two Qa-SNARE-SM complexes have shown that there are at least two different binding modes. In the first mode, the arch-shaped SM protein encloses and stabilizes the closed SNARE conformation (this conformation was studied in the Munc18-syntaxin-1 complex; see FIG. 1b for the closed conformation of syntaxin-1). In the second mode, the interaction is confined to a surface interaction with the $\mathrm{N}$-terminal end of the $\mathrm{SNARE}^{30}$. It has recently been proposed that the longin domain of the S. cerevisiae SNARE Ykt6 catalyses the palmitoylation of Vac8, a protein that is involved in vacuole fusion ${ }^{31}$. However, the profilin fold is shared by proteins that are unlikely to function as palmitoyltransferases, and there is no sequence similarity between longin domains and the known S. cerevisiae palmitoyltransferase families ${ }^{32}$.

Are the N-terminal domains needed for fusion? For some SNAREs, such as the S. cerevisiae Qa-SNARE Sso1, the $\mathrm{N}$-terminal domains are essential for cell viability ${ }^{33}$. However, when the N-terminal domain of Sso1 was replaced with the Qbc motifs of Sso9, a fused 'tandem SNARE' was created and function was restored ${ }^{34}$. These findings showed that, at least in this case, the $\mathrm{N}$-terminal domain is not needed for the recruitment of essential factors, which is in line with work showing that $\mathrm{N}$-terminal domains seem to be dispensable for fusion ${ }^{35}$.

\section{SNARE cycling in membrane fusion}

Shortly after the discovery that SNAREs are targets of NSF, it was proposed that fusion is mediated by the action of NSF on pre-assembled SNARE docking complexes ${ }^{8}$. As an alternative, some proposed that SNARE assembly might lead directly to fusion ${ }^{36,37}$. However, only after it was realized that NSF is not involved in fusion itself ${ }^{38}$, 


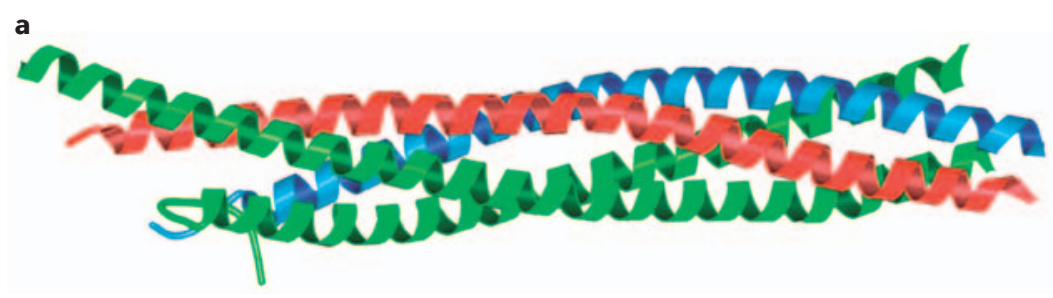

b
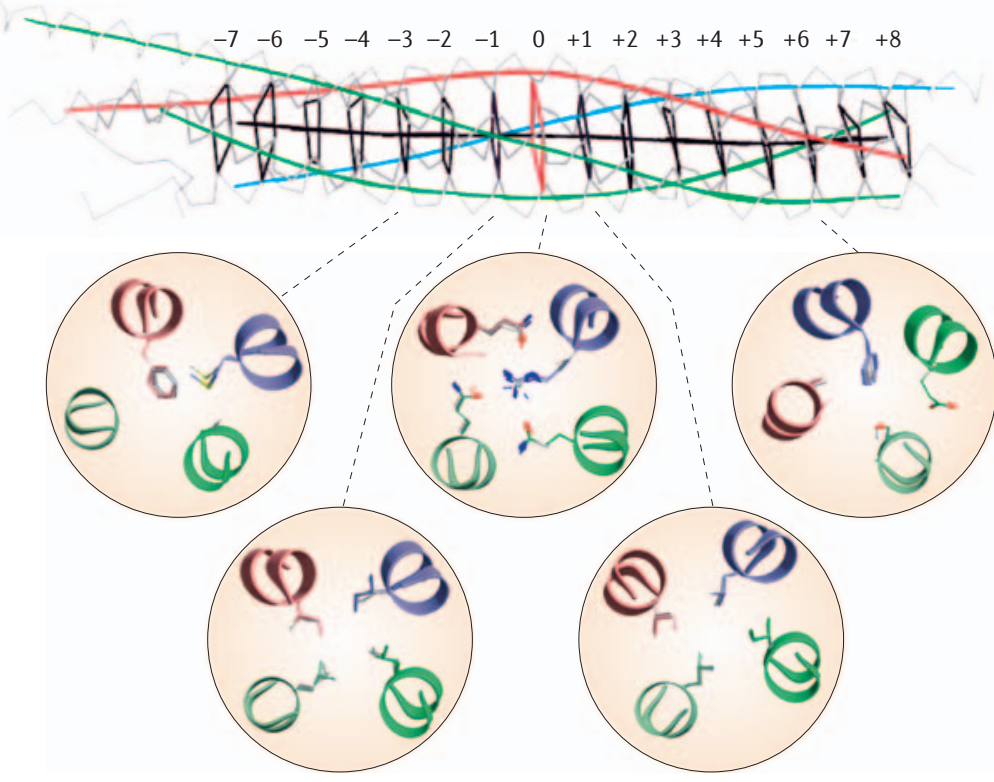

Figure 2 | SNARE core complexes. a | A crystal structure of the neuronal SNARE (soluble $\mathrm{N}$-ethylmaleimide-sensitive factor attachment protein receptor) core complex. This complex contains the SNARE motifs of syntaxin-1 (Qa; red), SNAP-25 (25-kDa synaptosome-associated protein; $\mathrm{Qb}$ and Qc; both green), and VAMP (vesicleassociated membrane protein)/synaptobrevin (R; blue). The C-terminal ends of the helices, which all point towards the membrane, are orientated to the right. Modified with permission from REF. 13 C Macmillan Magazines Ltd. b|A skeleton diagram that indicates the position of the central layers of interacting side chains (numbered) in the neuronal SNARE core complex. C $\alpha$ traces are shown in grey, the helical axes are highlighted by lines that are the same colour as the helices in part $\mathbf{a}$, and the superhelical axis is highlighted by a black line. The ' 0 ' layer is coloured red and all other layers are coloured black. Modified with permission from REF. 13 @ Macmillan Magazines Ltd. c | Overlays of individual layers, which are each shown contained in a shaded circle, from the neuronal SNARE core complex (grey) and the endosomal SNARE core complex (coloured). The endosomal SNARE core complex contains syntaxin-7 (Qa; red), VTI1b (Vps ten interacting-1b; Qb; light green), syntaxin-8 (Qc; dark green) and VAMP8 (R; blue). The three upper panels exemplify highly asymmetric layers that include the polar 0 layer and the -3 and +6 layers (the -3 and +6 layers contain conserved phenylalanines). The lower two panels show the hydrophobic layers that surround the 0 layer and are also highly conserved. Modified with permission from REF. 14 @ Macmillan Magazines Ltd.

\section{COPII}

(Coatomer protein complex-II). COPII assembles at the exit sites of the endoplasmic reticulum, which results in the formation of COPII-coated transport vesicles that are destined for the cis face of the Golgi apparatus or for an intermediate compartment. function as fusion catalysts. Although it is still controversial, this model (FIG. 3) has gained wide acceptance and will be discussed in detail below.

The status of 'free' SNAREs in membranes. The activity of NSF guarantees that free - that is, uncomplexed - SNAREs are constantly regenerated. However, the fact that they are free does not necessarily exclude the possibility that SNAREs interact with other proteins. Indeed, numerous diverse proteins have been shown to bind to specific SNAREs.

What might be the function of these binding proteins? First, they can be involved in SNARE sorting and recycling by associating with SNAREs during transport - for example, the transmembrane protein synaptophysin binds to the neuronal R-SNARE VAMP/synaptobrevin and can assist in its sorting ${ }^{41}$. Second, they can have a role in the recruitment of SNAREs into trafficking vesicles. For example, the ER-Golgi SNAREs Bet1, Sed5 and Sec22 were reported to bind to the Sec23-Sec24 subcomplex of the coatomer protein complex-II (COPII) coat during export from the $\mathrm{ER}^{42}$, and VAMP4 interacts with the adaptor protein-1 (AP1) complex at the trans-Golgi network ${ }^{43}$. Third, they can be involved in the formation of docking complexes. Examples include the early endosomal tethering factor EEA1 (early endosomal antigen-1), which binds to the endosomal SNAREs syntaxin-6 and syntaxin-13, and the $S$. cerevisiae multisubunit docking complexes HOPS/VpsC (homotypic fusion and vacuole protein sorting/class $\mathrm{C}$ vacuolar protein sorting (Vps) protein complex) and VFT (Vps fifty three), which bind to the Qc-SNAREs Vam3 and Tlg1 (t-SNARE affecting a late Golgi compartment-1), respectively ${ }^{44,45}$. Last, they can have a role in regulating the capability of SNARE motifs to enter SNARE complexes. For example, membrane-anchored VAMP/synaptobrevin was reported to be unable to form SNARE complexes due to the partial membrane insertion of the membrane-proximal part of its SNARE motif ${ }^{46,47}$. Activation by other proteins might therefore be required. Candidates for this role include the Rho GTPase Cdc42 (REF. 48) and the exocytic $\mathrm{Ca}^{2+}$ receptor synaptotagmin ${ }^{47}$. By contrast, the Q-SNAREs syntaxin-1 and SNAP-25 readily form core complexes with exogenous R-SNAREs in the plasma membrane, which indicates that these SNAREs are constitutively active ${ }^{49}$.

It must be stated, however, that for some of these proteins, and for many other proteins, the evidence that SNARE binding is specific and functionally relevant is not compelling. Free SNARE motifs are conformationally adaptable, which increases the chances of adsorptive and non-specific associations with other proteins in vitro. Furthermore, SNAREs are highly abundant - for example, syntaxin-1 and SNAP-25 each constitute $\sim 1 \%$ of the total brain protein ${ }^{50}$, which is probably greater than the sum of all of the ion channels and receptors that are in the neuronal plasma membrane. It is therefore unsurprising that almost every ion channel or receptor that has been studied has been reported to bind syntaxin-1 in a 'specific' manner. For the synaptic SNAREs alone, the number of reported binding proteins 


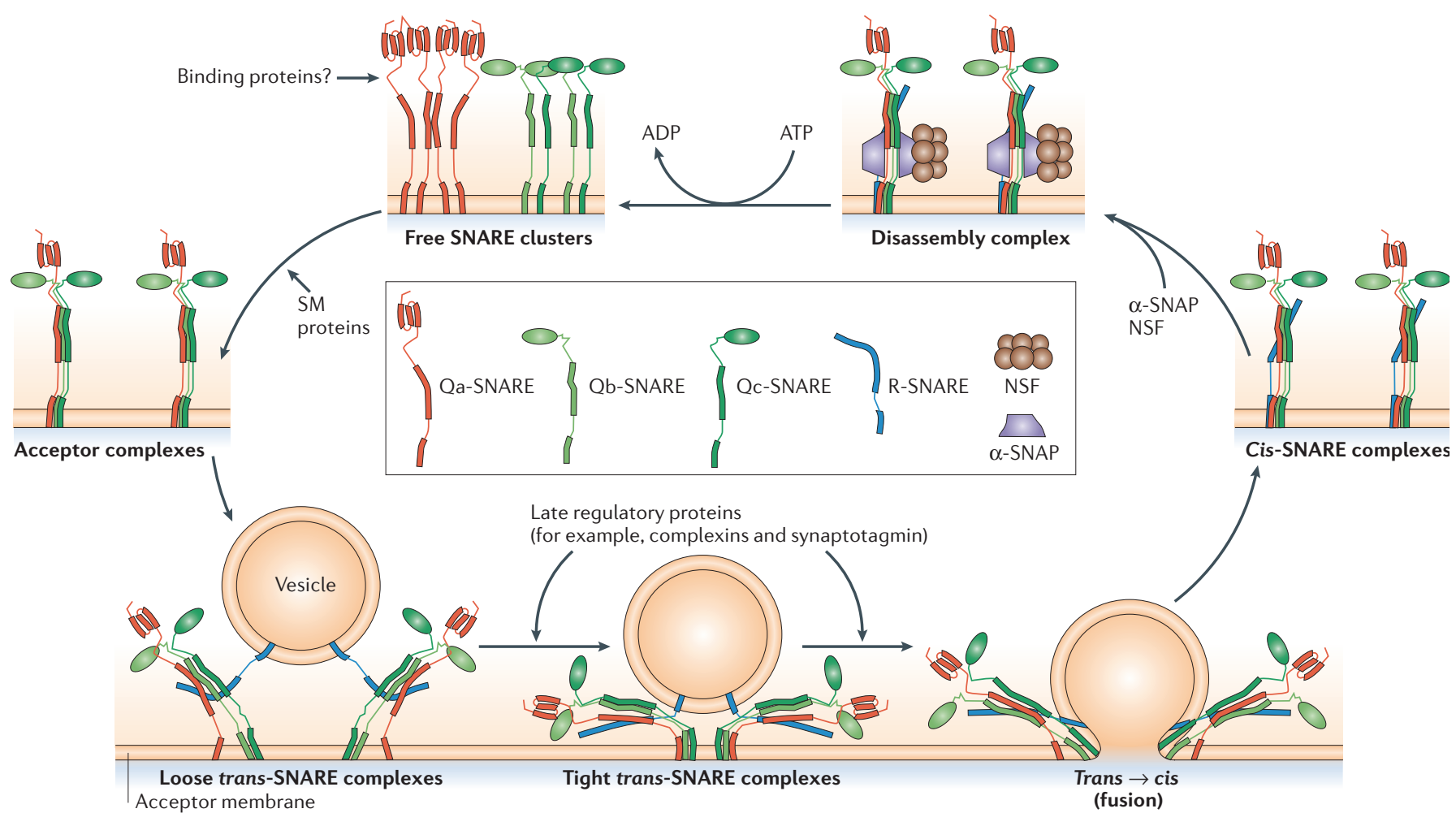

Figure 3 | The SNARE conformational cycle during vesicle docking and fusion. As an example, we consider three Q-SNAREs (Q-soluble $N$-ethylmaleimide-sensitive factor attachment protein receptors) on an acceptor membrane and an R-SNARE on a vesicle. Q-SNAREs, which are organized in clusters (top left), assemble into acceptor complexes, and this assembly process might require SM (Sec1/Munc18-related) proteins. Acceptor complexes interact with the vesicular R-SNAREs through the N-terminal end of the SNARE motifs, and this nucleates the formation of a four-helical transcomplex. Trans-complexes proceed from a loose state (in which only the N-terminal portion of the SNARE motifs are 'zipped up') to a tight state (in which the zippering process is mostly completed), and this is followed by the opening of the fusion pore. In regulated exocytosis, these transition states are controlled by late regulatory proteins that include complexins (small proteins that bind to the surface of SNARE complexes) and synaptotagmin (which is activated by an influx of calcium). During fusion, the strained trans-complex relaxes into a cis-configuration. Cis-complexes are disassembled by the AAA+ (ATPases associated with various cellular activities) protein NSF (N-ethylmaleimide-sensitive factor) together with SNAPs (soluble NSF attachment proteins) that function as cofactors. The R- and Q-SNAREs are then separated by sorting (for example, by endocytosis).

Adaptor protein-1 (AP1) complex

The AP1 complex, which is one of four structurally related protein complexes, forms a bridge between the clathrin coat and membrane components (cargo) during the formation of clathrincoated vesicles at the transGolgi network. is greater than 100. Most of the evidence is confined to qualitative approaches such as pull-downs and coimmunoprecipitations, which are notorious for yielding false positives with 'sticky' proteins. Quantitative and/or structural data about these presumed SNARE-targetprotein complexes are therefore largely missing. To validate these interactions, detailed structural information and a rigorous assessment of their in vivo relevance are required.

Plasma-membrane SNAREs are not uniformly distributed in the membrane, but are clustered in nanodomains, the stability of which depends on cholesterol ${ }^{51-53}$. The homomeric association of SNARE transmembrane domains has been reported, and this might contribute to cluster formation ${ }^{54}$. Secretory vesicles selectively dock and fuse at such clusters ${ }^{51}$. It remains to be seen whether cluster formation is a hallmark of all SNAREs or is a speciality of plasma membranes and other membranes that are rich in steroid lipids. Clustering achieves high local SNARE concentrations that might result in more efficient fusion.
Acceptor complexes: intermediates in the fusion pathway? How does the assembly of four unstructured SNAREs into a SNARE complex proceed? Detailed studies on exocytic S. cerevisiae and neuronal SNARE complexes in vitro have shown that assembly proceeds through a defined and partially helical Qabc intermediate ${ }^{5-57}$, the formation of which is rate limiting. Although it is not yet known whether other SNAREs form such intermediate acceptor complexes, it is probable that they represent a key step in the fusion pathway of all SNAREs - that is, it is likely that assembly is an ordered, sequential reaction rather than a random collision of four different SNARE motifs. Only when an acceptor scaffold is available in which the N-terminal ends of the SNARE motifs are structured is the final SNARE able to bind with biologically relevant kinetics and nucleate the zippering reaction.

Acceptor complexes are highly reactive and are therefore difficult to characterize. For example, in vitro, the neuronal acceptor complex readily recruits a second Qa-SNARE, which results in a 'dead-end' Qaabc complex. 
This interaction prevents the binding of the R-SNARE and seems to be primarily responsible for the slow assembly kinetics of core complexes ${ }^{56}$. However, it is conceivable that acceptor complexes are stabilized by other factors, and SM proteins are prime candidates. For example, the SM protein Sly1 (suppressor of loss of Ypt1 protein-1) is thought to be involved in the formation of acceptor complexes of SNAREs that function in ER-Golgi transport $^{58}$. Another example is provided by the SM protein Vps33, which - in association with other proteins of the HOPS/VpsC complex - is thought to prepare SNAREs for the homotypic fusion of $S$. cerevisiae vacuoles (see, for example, REF. 45). Also, the S. cerevisiae Qa-SNARE Tlg2 cannot form SNARE complexes in strains that lack the SM protein Vps45 (REF. 59). However, work on other SM proteins, such as Munc18, indicates that they function either earlier in the pathway ${ }^{60-62}$ or after the formation of trans-SNARE complexes ${ }^{63}$, and, so far, the structures of Qa-SNARE-SM-protein complexes are difficult to reconcile with a function for SM proteins in acceptorcomplex stabilization ${ }^{30}$. There is therefore as yet no coherent molecular model for the role of SM proteins.

There is, so far, only scant and indirect information regarding whether such acceptor complexes exist in native membranes or in intact cells and whether they always have a Qabc composition. For the SNAREs that mediate neuronal exocytosis, the presence of Qabc acceptor complexes in the plasma membrane has been invoked from both SNARE-binding studies and live-cell imaging $^{49,64}$. However, a kinetic analysis of exocytosis in the presence of various combinations of soluble SNARE motifs indicated that, prior to fusion, VAMP/synaptobrevin and SNAP-25 become associated before syntaxin binds ${ }^{65}$. Another example of a putative acceptor complex has recently been described for the $S$. cerevisiae SNAREs Ufe1 (Qa), Sec20 (Qb) and Use1 (unconventional SNARE in the ER protein-1; Qc), which function in the fusion of retrograde transport vesicles with the ER. A complex of these SNAREs forms a 1/1 association of unusual stability with a multiprotein complex termed the Dsl complex (this complex is named after one of its constituents, Dsl1, which is an ER-localized peripheral membrane protein $)^{66}$. Interestingly, the C-terminal domain of Dsl1 resembles R-SNARE motifs, which indicates that it might occupy the R-SNARE-binding site of the acceptor complex ${ }^{66}$. Moreover, the neuron-specific protein tomosyn, which has a C-terminal R-SNARE motif, is thought to regulate neuronal Q-SNAREs by a similar mechanism ${ }^{67-69}$. More proteins with partial or complete SNARE motifs have recently been identified $^{70,71}$. It is therefore conceivable that at least some acceptor complexes are stabilized, or regulated, by such 'pseudo-SNARE' proteins. These protein might function as a 'place holder' for the SNARE that will be contributed by the opposing membrane.

Formation and maintenance of trans-SNARE complexes. A central tenet of the zippering hypothesis of SNARE function is that for fusion to proceed, SNAREs must assemble in a trans configuration, with at least one SNARE that has a transmembrane domain being contributed by each of the fusing membranes (FIG. 3). Assembly starts at the $\mathrm{N}$ termini of the SNARE motifs and then proceeds in a zipper-like fashion towards the C-terminal membrane anchors. As a result, mechanical force is exerted on the membranes, which might overcome the energy barrier for fusion.

Abundant data show that for fusion to occur, SNAREs must reside in both of the membranes that are undergoing fusion ${ }^{72}$. Furthermore, mutating amino acids in the centre of the four-helix bundle generally impairs fusion ${ }^{15,73}$. However, although trans-SNARE complexes are widely thought to be essential intermediates in the fusion pathway, direct evidence has been difficult to obtain. Trans-SNARE complexes are transient structures, and attempts to isolate them involve the detergent solubilization of membranes, which results in their immediate conversion from trans to cis forms.

The best, albeit still indirect, evidence for the existence of trans-SNARE intermediates comes from the study of regulated exocytosis in neurons and neuroendocrine cells (see REF. 74 for a recent review). Unlike other intracellular fusion events, exocytosis is regulated at a late step before membrane merger. It is probable that metastable trans-SNARE complexes persist for some time until exocytosis is triggered by $\mathrm{Ca}^{2+}$ influx. In chromaffin cells, kinetically distinct pools of vesicles, which represent sequential steps along the pathway to exocytosis, can be distinguished by electrophysiological methods. SNARE assembly has been manipulated in this system by various means. Approaches include the use of anti-SNARE antibodies and SNARE-cleaving toxins, and the expression of SNAP-25 and VAMP/synaptobrevin variants in cells from knockout mice that lacked these proteins ${ }^{74,75}$. Any manipulations that are expected to impair the zippering of SNAREs reduce exocytosis, and some of the data are best explained by a model in which there is an equilibrium between free and partially zippered trans-SNARE complexes before exocytosis.

As is the case for every other intermediate of the SNARE cycle, trans-SNARE complexes are subject to regulation by other proteins (FIG. 3). In neuronal exocytosis, synaptotagmin I - a Ca ${ }^{2+}$-sensing transmembrane protein of synaptic vesicles - is essential for fast, $\mathrm{Ca}^{2+}$-triggered fusion (reviewed in REFS 76,77). Synaptotagmin contains two $\mathrm{Ca}^{2+}$-binding $\mathrm{C} 2$ domains (conserved region-2 of protein kinase $\mathrm{C}$ domains) that interact both with SNAREs and with acidic phospholipids in $\mathrm{Ca}^{2+}$-dependent manner. Following $\mathrm{Ca}^{2+}$ binding, synaptotagmin is thought to promote fusion, although it is still unclear to what extent this is caused by SNARE or phospholipid binding by the C2 domains. So far, 14 different synaptotagmins have been identified in mammals, some of which have a widespread tissue distribution. This indicates that synaptotagmins might have a general role in regulating SNARE-mediated fusion ${ }^{78}$. A second example of SNARE-binding proteins are the complexins. Complexins are small helical proteins that bind to the surface of SNARE complexes, and they are also involved in the $\mathrm{Ca}^{2+}$-dependent triggering step of neuronal exocytosis ${ }^{79}$. Intriguingly, a structure of a complexin-SNARE complex indicates that complexins 


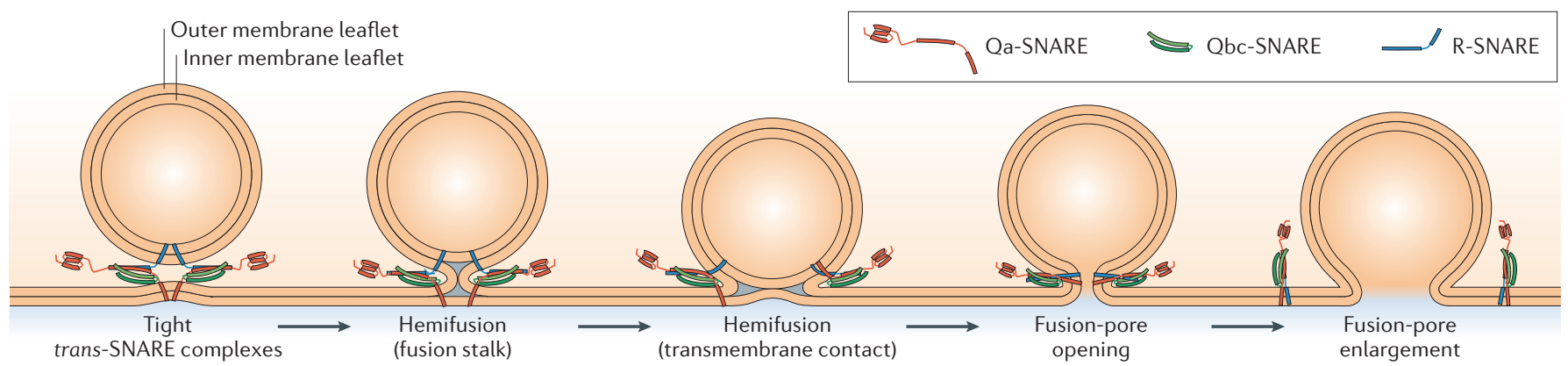

Figure 4 | Hypothetical transition states in SNARE-mediated fusion according to the stalk hypothesis. The stalk hypothesis is a macroscopic theory that treats membranes as bendable sheets and largely ignores local fluctuations of lipids $^{82}$. Hemifusion is defined as the state in which the outer membrane leaflets are already continuous, but no aqueous connection has formed. In the non-bilayer transition states, the linker regions that connect the SNARE (soluble $\mathrm{N}$-ethylmaleimide-sensitive factor attachment protein receptor) motifs to the transmembrane domain must either be bent (possibly strained), or the C-terminal ends must be pulled into the hydrophobic core of the bilayer, which would allow the formation of cis complexes before fusion is complete (as is indicated on the right side of the "Hemifusion (transmembrane contact)' SNARE complex). The idea that the C-terminal end is pulled into the hydrophobic core of the bilayer is supported by the fact that, with few exceptions, SNAREs only contain a few moderately hydrophilic residues that are C-terminal of the transmembrane domain, which might facilitate the immersion of this region into the hydrophobic core of the transition states. In this case, the assembly of at least some SNARE complexes might therefore be complete before fusion-pore opening. The hydrophobic voids that are invoked by the stalk hypothesis are highlighted in grey. The SNAREs, the membrane thickness and the vesicles are drawn approximately to scale.

can bind to partially assembled SNARE complexes ${ }^{80}$. It is currently not known whether other SNARE complexes are subject to similar regulatory mechanisms.

Fusion. During fusion, opposing membranes that are in contact proceed through a series of intermediates. As a result, an aqueous fusion pore is formed that connects the distal leaflets of the fusing membranes while maintaining a lipidic seal between the distal and the proximal leaflets during the reaction. An intuitive and physically well founded model is provided by the stalk hypothesis that, although not without challenges, describes fusion as an ordered sequence of lipidic non-bilayer transition states (FIG. 4; reviewed in REFS 81,82).

What is the role of SNAREs in lipid merger? The best-supported model indicates that SNARE assembly exerts a mechanical force on membranes, which directly causes fusion. The model implies that the linkers between the transmembrane domains and the helical bundle of SNARE motifs are stiff. Therefore, straining these linkers transmits energy onto membranes, bending them and/or disturbing the hydrophilic-hydrophobic boundary (FIG. 4). As a result, the opposing membranes are not only pressed against each other but they are also deformed, which facilitates the formation of fusion stalks. This model gives rise to certain predictions, some of which have been be verified experimentally. First, syntaxin assumes an upright orientation when it is reconstituted into planar lipid bilay$\mathrm{ers}^{83}$, in agreement with the prediction of a rigid linker region, which was made on the basis of molecular dynamics simulations ${ }^{84}$. Second, fusion is blocked if a SNARE transmembrane domain is replaced by a more flexible lipid anchor, or if extra amino acids are inserted between the SNARE motif and the transmembrane domain, both in biological fusion reactions ${ }^{35,85}$ and following the reconstitution of SNAREs into proteoliposomes ${ }^{86}$.
Although the force model is intuitively satisfying, many details are still unknown. For example, it is unclear how many SNARE complexes are needed for a single fusion event. Estimates range between 3 and 15 (REF. 87), although it is difficult to envisage how 15 SNAREs could fit around a fusion pore with a diameter of only few nanometres. Similarly, the nature of the intermediate stages is unclear, although there has been some recent progress (FIG. 4). SNARE-mediated fusion in both native membranes and proteoliposomes can be arrested at a hemifusion state $^{88-90}$. Hemifusion is experimentally defined as a state in which the lipids of the proximal leaflets are already exchanging, but an aqueous connection between the structures that are undergoing fusion has not yet formed (FIG. 4). It remains to be determined whether hemifusion is a genuine intermediate of the reaction, or whether it represents a trapped 'dead-end' reaction that leads to the expansion of a hemifusion diaphragm, as has been discussed for viral fusion proteins (for a review, see REF. 82).

Alternative ideas have also been discussed. For example, amphiphilic regions of the SNAREs might perturb the hydrophilic-hydrophobic boundary, leading to the formation of non-bilayer transition states without the use of mechanical force ${ }^{91}$. Moreover, isolated SNARE transmembrane domains were shown to be capable of driving liposome fusion ${ }^{92}$. Amphiphilic peptides, such as viral fusion peptides, are potent fusogens in vitro. Although these models fail to explain why only QabcR complexes are fusion competent, it is conceivable that a surface-perturbing function of the membrane-proximal regions or transmembrane domains of SNAREs contributes to membrane merger once a state of close apposition has been reached.

In summary, a large body of evidence supports the view that SNAREs function as fusion catalysts that not only provide the energy for the fusion reaction, but 
that also bring fusion to completion. However, some researchers have suggested that further proteins are required that operate downstream of SNAREs. The vacuolar $\mathrm{H}^{+}$-ATPase - a highly conserved enzyme that is both structurally and mechanistically related to the mitochondrial ATP synthase ${ }^{93}$ - was proposed to function after SNARE assembly in the fusion pathway ${ }^{94,95}$. In particular, it was proposed that fusion is not mediated by SNAREs, but by the $\mathrm{V}_{0}$-subunits of the vacuolar ATPase, with the role of SNAREs being confined to membrane docking ${ }^{96,97}$. According to this view, the proteolipid $\mathrm{V}_{0}$-ring of the vacuolar $\mathrm{H}^{+}$-ATPase can function as a conserved fusion channel. During fusion, two proteolipid rings connect in trans and open to form an aqueous fusion pore. Up to this point, the mechanism resembles that of connexins, which form gap-junction channels between cells, but then the pore must enlarge by further ring opening and subunit separation, which is associated with a break of the trans connection. We find it hard to understand how the channel connects the two membranes, how the lipids invade the subunit structure and how the assembled double ring is disassembled, and these are all issues that are well explained by the model of SNARE-mediated fusion. So far, the evidence for a role for the vacuolar $\mathrm{H}^{+}$-ATPase in membrane fusion is indirect, and no mechanistic molecular model is available that agrees with biochemical and biophysical principles.

Disassembly and recycling. After fusion, SNARE complexes are transformed from a trans- to cis-configuration, in which all of the SNAREs of a complex reside together in the resulting fused membrane. This configuration represents the low point in terms of potential energy, and it is thought that such complexes are biologically inactive until the complex is dissociated. Disassembly requires considerable metabolic energy, and this energy is provided by $\mathrm{NSF}^{8}$, a hexameric member of the AAA+-protein family. Many AAA+ proteins operate as 'unfoldases' that disentangle protein complexes and aggregates $^{98}$. On its own, NSF does not interact with SNARE complexes. It requires cofactors that are known as SNAPs (soluble NSF attachment proteins) that bind to the surface of the SNARE complex (FIG. 3). Both NSF and SNAPs - which include three isoforms that are termed $\alpha-, \beta$ - and $\gamma$-SNAP - are highly conserved and seem to function on all SNARE complexes.

Despite a wealth of structural information about the components of the disassembly reaction, it is still unclear how NSF manages to disassemble complexes that are stable under conditions as extreme as $80^{\circ} \mathrm{C}, 8 \mathrm{M}$ urea or $2 \% \mathrm{SDS}^{99}$. Three SNAP molecules bind to the middle of the SNARE complex ${ }^{100}$, at a position that is approximately centred around the hydrophilic 0 layer (FIG. 2). Intriguingly, by systematically mutating amino acids in the various layers, the 0 layer was independently identified as being crucial for dissociation ${ }^{101}$. Bound $\alpha$-SNAPs, in turn, recruit and activate NSF. NSF has three distinct domains: two homologous $\mathrm{D}$ domains that contain canonical ATP-binding sites (D2 is catalytically inactive and mediates hexamerization); and an
$\mathrm{N}$ domain that undergoes large conformational changes during the catalytic cycle (for a review, see REF. 98). How exactly NSF operates is unknown. Although the substrate is known to traverse the central hole of the hexameric ring in the AAA+ protein CIpA, the assembled SNARE complex is too large to pass through the central hole of NSF. It has recently been shown for another AAA+ protein, CIpX, that ATP hydrolysis and the rebinding of ClpX to its substrate is not synchronized between the ClpX subunits ${ }^{102}$. This independent functioning of the subunits might explain how AAA+ proteins, despite their hexameric symmetry, can dissociate asymmetric protein complexes.

The complete dissociation of SNARE complexes might involve several catalytic cycles of NSF with numerous ATP-hydrolysis events, which would provide more than sufficient energy for disassembling SNAREs. Intriguingly, the NSF-SNAP system not only operates on SNARE core complexes but can also dissociate at least some of the off-pathway complexes ${ }^{103,104}$. Presumably, this provides a biological safeguard mechanism that protects cells against the accumulation of dead-end by-products that are derived from the highly reactive SNAREs.

SNARE disassembly by NSF is crucial for maintaining fusion competence in the secretory pathway. Indeed, many in vitro fusion reactions show a strict dependence on active NSF, and models have been proposed in which NSF-mediated disassembly defines an intermediate step in the ordered sequence of reactions that lead to fusion $^{105,106}$. However, whereas NSF-driven disassembly might be rate-limiting in vitro, this is probably not the case in intact cells. The study of temperature-sensitive NSF mutants in S. cerevisiae and Drosophila melanogaster has shown that SNARE complexes only accumulate at the restrictive temperature - that is, when NSF is inactive ${ }^{63,107,108}$. Apparently, the continuous activity of the NSF-SNAP system ensures that core complexes are rapidly disassembled under normal steady-state conditions.

\section{SNARE localization and specificity}

Eukaryotic cells contain many SNAREs, with numerous members in each subfamily ${ }^{2}$. Although some SNAREs seem to function in only one intracellular fusion step and to interact with only one set of partner SNAREs, others are less specialized, which provides a healthy mix of robustness and flexibility for intracellular fusion reactions.

Localization and sorting of SNAREs. For SNAREs to function in specific intracellular fusion steps, there must be sorting mechanisms that ensure that each intracellular membrane is equipped with the appropriate set of SNARE proteins. Indeed, many SNAREs reside predominantly, or even selectively, in specific subcellular compartments. Examples include syntaxin-1, syntaxin-2, syntaxin-4, SNAP-23 and SNAP-25 at the plasma membrane, as well as VAMP/synaptobrevin on synaptic and neurosecretory vesicles, and syntaxin-5 and VAMP4 in the Golgi apparatus (reviewed in REF. 2). However, it is clear that 
a S. cerevisiae
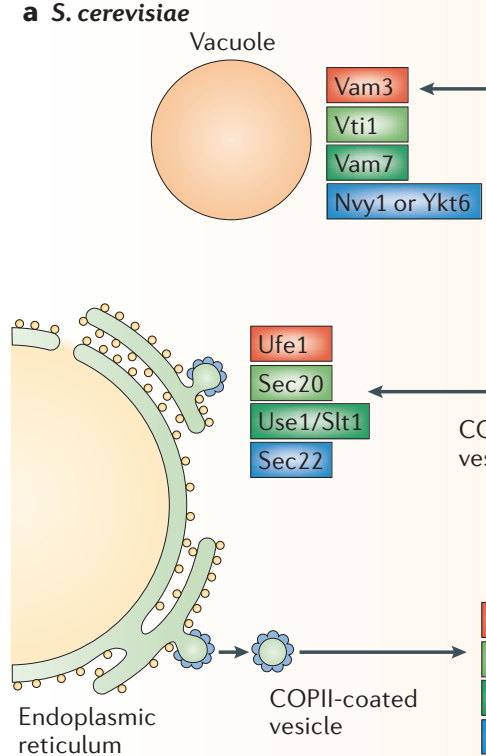

reticulum
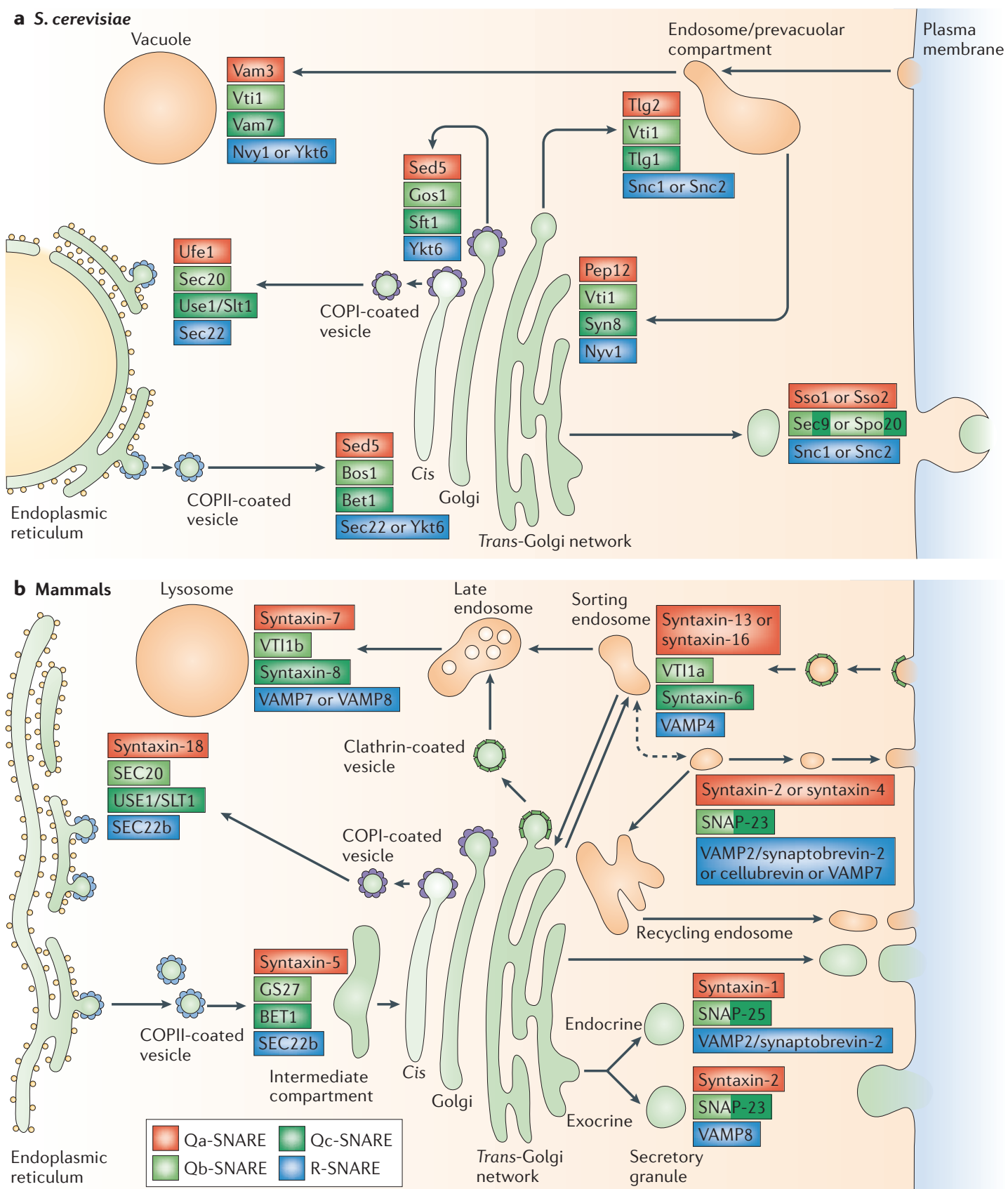

Figure 5 | The assignment of SNAREs to intracellular membrane-trafficking pathways. a | A full complement of QabcR-SNAREs can be assigned to all of the fusion events in the secretory pathway of Saccharomyces cerevisiae. The biosynthetic route begins at the endoplasmic reticulum (ER) and leads, through coatomer protein complex-II (COPII)coated vesicles, to the Golgi complex. From the trans-Golgi network, transport vesicles are directed to the growth pole (bud) where they fuse with the plasma membrane. A second route leads to the prevacuolar compartment. The retrograde route leads from the plasma membrane, through the endosome/prevacuolar compartment, to the vacuole (this is simplified). Note that the R-SNAREs Nvy1 and Ykt6 can substitute for each other in vacuole fusion, and that the R-SNAREs Sec22 and Ykt6 can substitute for each other in the fusion of ER-derived transport vesicles with the cis-Golgi. Snc1 and Snc2, as well as Sso1 and Sso2, are homologous isoforms, and Spo20 substitutes for Sec9 during spore formation. b | The assignment of SNAREs to trafficking pathways in mammalian cells. For many of the fusion reactions, SNAREs have not yet been unambiguously identified. The mammalian trafficking pathways resemble those of S. cerevisiae, but further intermediates are involved. Also shown are the specialized storage organelles for regulated exocytosis. The dashed double-headed arrow indicates homotypic fusion between two sorting endosomes. See REF. 1 for an overview of eukaryotic trafficking pathways. COPI, coatomer protein complex-l; Gos1, Golgi SNARE protein-1; GS27, Golgi SNARE of 27 kDa; SNAP-23, 23-kDa synaptosome-associated protein; SNAP-25, 25-kDa synaptosome-associated protein; SNARE, soluble $\mathrm{N}$-ethylmaleimide-sensitive factor attachment protein receptor; Spo20, sporulation-specific protein-20; Syn8, syntaxin-8; Tlg, t-SNARE affecting a late Golgi compartment; Use1/USE1, unconventional SNARE in the ER protein-1; Vam, vacuole membrane; VAMP, vesicle-associated membrane protein; Vti1/VTI1, Vps ten interacting-1. 


\section{Box 1 |The fusion of liposomes that have been reconstituted with SNAREs}

The ultimate goal for research into supramolecular machines is to be able to reconstitute them in an active form from purified components. Indeed, liposomes that have been reconstituted with purified SNARE (soluble $\mathrm{N}$-ethylmaleimidesensitive factor attachment protein receptor) proteins undergo fusion in a manner that shares certain characteristics with biological fusion reactions ${ }^{129}$. For example, this liposome fusion involves the formation of SNARE complexes, is sensitive to SNARE inhibitors such as the botulinum and tetanus neurotoxins, and requires the presence of at least one SNARE with a transmembrane domain (rather than a lipid anchor) in each membrane ${ }^{86}$.

However, other essential features of biological fusion reactions have not yet been reproduced in liposomes. The liposome fusion rates are slow, and this is not because the docking is rate limiting ${ }^{130}$. Furthermore, it has proven difficult to reconstitute regulatory steps. For example, a cytoplasmic fragment of synaptotagmin induced a moderate, $\mathrm{Ca}^{2+}-$ dependent acceleration of the fusion between liposomes that contained neuronal SNAREs ${ }^{131}$, but no stimulation was measured when the intact protein was used instead ${ }^{131,132}$. What might be the reason for these problems? In addition to the difficulty of controlling SNARE conformations in vitro, the biophysical properties of artificial vesicles might differ from those of biological membranes. Small unilamellar liposomes can easily be induced to fuse effectively by small amphiphilic molecules, and they can become leaky and fusion prone at high protein/phospholipid ratios ${ }^{22}$. The activation energy for fusion is therefore not overly high, which is in contrast to other in vitro reactions that are catalysed by supramolecular machines. Furthermore, the fusion kinetics of SNARE-containing liposomes strongly depend on experimental conditions ${ }^{133}$. This problem is particularly prevalent for planar membranes that are prone to undergo fusion with liposomes in a non-specific manner. For example, vesicles reconstituted with the neuronal R-SNARE VAMP (vesicle-associated membrane protein; also known as synaptobrevin) readily fused with flat membranes containing the Qa-SNARE syntaxin regardless of whether the Qbc-SNARE SNAP-25 (25-kDa synaptosome-associated protein) was present ${ }^{134}$ or not ${ }^{135,136}$. Apparently, the weak interaction between syntaxin and VAMP/synaptobrevin suffices to induce fusion, which makes it difficult to discern such artefacts from biologically meaningful reactions. It remains to be established to what extent the fusion pathway and the transition states of such artificial systems resemble genuine biological fusion reactions.

after completing most fusion reactions - including all heterotypic fusions in which a small trafficking vesicle fuses with a large acceptor compartment — some SNAREs need to be returned to their donor compartment by intracellular membrane trafficking. Consequently, SNAREs reside not only in the organelle for which they mediate fusion, but they also reside in the membranes of the organelles that are part of their recycling pathway. For example, SNAREs that are involved in trafficking between the ER and the Golgi are found in both of these compartments and in the intermediate trafficking vesicles (see, for example, REFS 109,110). Some other SNAREs have an even more widespread distribution, including those that are involved in endosomal trafficking ${ }^{111}$. Localization is therefore dependent on the steady state between SNARE biosynthesis, fusion and recycling, and it is not possible to deduce in what fusion step a given SNARE functions from its localization alone.

Undoubtedly, accessory proteins that bind to free SNAREs have a role in SNARE sorting and localization. However, although the cytoplasmic domains of SNAREs are essential for their correct sorting ${ }^{112,113}$, with a few specialized exceptions (see, for example, REF. 43), no defined sorting signals have been identified. An almost bewildering number of SNARE mutations cause missorting. Interestingly, many of these mutations are in the SNARE motif and often include the amino-acid side chains of the central layers ${ }^{114}$. It is therefore probable that sorting also depends on SNARE conformation, but we do not know whether sorting occurs only when a SNARE is free or whether SNARE complexes are also sorted. In fact, many 'sorting' mutations might change the intracellular distribution by changing the relative distribution of the affected SNAREs between the conformational pools, for example, by impairing assembly or disassembly.
Specificity of SNAREs. The secretory pathway of eukaryotic cells consists of well organized routes that connect intracellular organelles by vesicular traffic (FIG. 5). Membrane fusion is the final and irreversible step of each trafficking route. Under normal homeostatic conditions, only the appropriate organelles fuse with each other, which raises the questions of at what level and by which mechanisms are the specificity of fusion regulated? Although it has become clear that Ras-related small GTPases of the Rab/Ypt (yeast protein transport) family are instrumental in controlling the specificity of the initial contact (tethering and docking) between the fusion partners (reviewed in REF. 115), to what extent SNAREs contribute to fusion specificity is still debated.

In vitro experiments have shown that SNAREs assemble rather promiscuously into core complexes ${ }^{116,117}$. Although certain combinations are more stable than others, such promiscuity is not overly surprising considering the extraordinary degree of structural conservation between different SNARE complexes. By contrast, a high degree of specificity has been observed in a comprehensive analysis of liposome fusion that is mediated by S. cerevisiae SNAREs ${ }^{9,118,119}$. However, efficient liposome fusion has recently been observed between endosomal and exocytic SNAREs, which do not interact physiologically with each other ${ }^{120}$ (BOX 1). It is therefore conceivable that many of the SNARE combinations that form stable QabcR complexes in vitro fuse liposomes, although restrictions might apply with respect to the distribution of the individual SNAREs between membranes in vivo.

On the other hand, SNAREs are undoubtedly specialized for individual intracellular fusion reactions. In S. cerevisiae, specific sets of SNAREs can be assigned to most fusion steps (FIG. 5a), and candidates have been proposed for many of the fusion steps in mammalian 
cells (FIG. 5b). A picture is emerging regarding which SNAREs have acquired specificity during evolution, but the degree to which specificity has been achieved seems variable. In many cases, the deletion of an individual SNARE does not prevent fusion, but rather impairs it, which can result in surprisingly mild phenotypes. These observations indicate that SNAREs can functionally replace each other to a certain extent. For example, in S. cerevisiae ER-Golgi traffic, Sec22 and Ykt6 can substitute for each other as the R-SNARE of a SNARE complex ${ }^{121}$. Similarly, SNAP-25 and SNAP-23, as well as VAMP2/synaptobrevin-2 and cellubrevin, are capable of substituting for each other to a varying degree in the regulated exocytosis of chromaffin cells ${ }^{75,122}$. Conversely, individual SNAREs can operate in more than one fusion step that involve different SNARE partners. Examples include Ykt6 (REF. 123), Sed5 (REF. 124) and VAMP8 (also known as endobrevin). The situation for VAMP8/endobrevin is particularly noteworthy because the protein functions not only in late-endosome fusion, but also in exocytosis in the exocrine pancreas ${ }^{125,126}$. Soluble SNAREs compete with endogenous SNAREs in biological fusion reactions, but, again, specificity is not absolute. For example, in neuronal exocytosis, the endogenous R-SNARE VAMP/synaptobrevin is not only inhibited by its own soluble fragment, but also by other R-SNAREs, although the potency of the effect varies ${ }^{127}$.

Considering the relative promiscuity of SNARE pairing in vitro, further factors need to be invoked to ensure specificity. In particular, the question arises of how a cell manages to select one set of SNAREs for an upcoming fusion step, while silencing others that might be present in the same membrane. For example, VAMP/synaptobrevin functions in neuronal exocytosis but not in the fusion of early endosomes ${ }^{128}$, even though it recycles through this compartment, can form in vitro complexes with endosomal SNAREs ${ }^{116}$ and can mediate liposome fusion with endosomal SNAREs ${ }^{120}$. Although there is currently no general answer to this question, it is conceivable that the formation of acceptor complexes is tightly regulated and therefore contributes to SNARE specificity. Alternatively, the main specificity checkpoint might be at the level of tethering - that is, the first molecular link between the vesicle and the target membrane. Tethering complexes might operate at a distance of up to $50 \mathrm{~nm}$ between the membranes, whereas the earliest stage of SNARE pairing cannot occur until the distance is below $25 \mathrm{~nm}$. The organization of the membrane compartments of eukaryotic cells is therefore likely to arise from numerous layers of regulation, many of which probably occur before the formation of the SNARE complexes that catalyse the final and irreversible step.

\section{Conclusions and outlook}

Intracellular fusion reactions are mediated by supramolecular complexes that are assembled on demand and disassembled when the task is complete. Of the many proteins that are involved in these complexes, SNAREs represent the core of the fusion engine - that is, they operate similarly to 'blue collar' workers who get the job done. Although they are small and have a simple domain structure, SNAREs carry out a conformationally controlled reaction cycle of high complexity that is still not completely understood, despite recent progress. Understanding of all of the steps of the cycle is a prerequisite for elucidating how SNAREs are regulated. For each potential SNARE-controlling protein, where it functions in the conformational cycle of SNAREs and which of the partial reactions it influences need to be established. For most of the proposed regulators, this has not yet been achieved. However, SNARE regulation is not only mediated by proteins that bind to SNAREs, but also by a panoply of protein kinases and phosphatases, and by other signalling proteins that can control SNAREs either directly or indirectly by influencing the proteins that function on SNAREs. Understanding these layers of regulation will be one of the main challenges for the coming years.
Bonifacino, J S. \& Glick, B. S. The mechanisms of vesicle budding and fusion. Cell 116, 153-166 (2004).

2. Hong, W. SNAREs and traffic. Biochim. Biophys. Acta 1744, 120-144 (2005)

3. Fasshauer, D. Structural insights into the SNARE mechanism. Biochim. Biophys. Acta 1641, 87-97 (2003).

4. Brunger, A. T. Structure and function of SNARE and SNARE-interacting proteins. Q. Rev. Biophys. 38, 1-47 (2005).

5. Rossi, V. et al. Longins and their longin domains: regulated SNAREs and multifunctional SNARE regulators. Trends Biochem. Sci. 29, 682-688 (2004).

6. McNew, J. A. et al. Ykt6p, a prenylated SNARE essential for endoplasmic reticulum-Golgi transport. J. Biol. Chem. 272, 17776-17783 (1997).

7. Valdez-Taubas, J. \& Pelham, H. Swf1-dependent palmitoylation of the SNARE TIg 1 prevents its ubiquitination and degradation. EMBO J. 24, 2524-2532 (2005).

8. Söllner, T., Bennett, M. K., Whiteheart, S. W., Scheller, R. H. \& Rothman, J. E. A protein assemblydisassembly pathway in vitro that may correspond to sequential steps of synaptic vesicle docking, activation, and fusion. Cell 75, 409-418 (1993). Shows that ATP cleavage by NSF is associated with the disassembly of a pre-existing SNARE complex, providing the first direct evidence for the existence of an assembly-disassembly cycle that underlies membrane fusion.

9. McNew, J. A. et al. Compartmental specificity of cellular membrane fusion encoded in SNARE proteins. Nature 407, 153-159 (2000)

10. Lewis, M. J., Rayner, J. C. \& Pelham, H. R. A novel SNARE complex implicated in vesicle fusion with the endoplasmic reticulum. EMBO J. 16, 3017-3024 (1997).

11. Dilcher $M$ et al. Use1 $\mathrm{p}$ is a yeast SNARE protein required for retrograde traffic to the ER. EMBO J. 22 3664-3674 (2003)

12. Burri, L. et al. A SNARE required for retrograde transport to the endoplasmic reticulum. Proc. Nat Acad. Sci. USA 100, 9873-9877 (2003).

13. Sutton, R. B., Fasshauer, D., Jahn, R. \& Brunger, A. T. Crystal structure of a SNARE complex involved in synaptic exocytosis at $2.4 \AA$ resolution. Nature 395 , 347-353 (1998).

The first high-resolution structure of a SNARE complex.

14. Antonin, W., Fasshauer, D., Becker, S., Jahn, R. \& Schneider, T. R. Crystal structure of the endosomal SNARE complex reveals common structural principles of all SNAREs. Nature Struct. Biol. 9, 107-111 (2002).

15. Fasshauer, D., Sutton, R. B., Brunger, A. T. \& Jahn, R. Conserved structural features of the synaptic fusion complex: SNARE proteins reclassified as Q- and
R-SNARES. Proc Natl Acad Sci. USA 95, 15781-15786 (1998)

16. Bock, J. B., Matern, H. T., Peden, A. A. \& Scheller, R. H A genomic perspective on membrane compartment organization. Nature 409, 839-841 (2001).

17. Misura, K. M. Scheller, R. H. \& Weis, W. I. Selfassociation of the $\mathrm{H} 3$ region of syntaxin $1 \mathrm{~A}$. Implications for intermediates in SNARE complex assembly. J. Biol. Chem. 276, 13273-13282 (2001).

18. Misura, K. M., Gonzalez, L. C. Jr, May, A. P. Scheller, R. H. \& Weis, W. I. Crystal structure and biophysical properties of a complex between the $\mathrm{N}$-terminal SNARE region of SNAP25 and syntaxin 1a. J. Biol. Chem. 276, 41301-41309 (2001).

19. Margittai, M., Fasshauer, D., Pabst, S., Jahn, R. \& Langen, R. Homo- and heterooligomeric SNARE complexes studied by site-directed spin labeling. J. Biol. Chem. 276, 13169-13177 (2001).

20. Zhang, F., Chen, Y., Kweon, D. H., Kim, C. S. \& Shin, Y. K. The four-helix bundle of the neuronal target membrane SNARE complex is neither disordered in the middle nor uncoiled at the C-terminal region. J. Biol. Chem. 277 24294-24298 (2002).

21. Weninger, K., Bowen, M. E., Chu, S. \& Brunger, A. T. Single-molecule studies of SNARE complex assembly reveal parallel and antiparallel configurations. Proc. Natl Acad. Sci. USA 100, 14800-14805 (2003). 
22. Dennison, S. M., Bowen, M. E., Brunger, A. T. \& Lentz, B. Neuronal SNAREs do not trigger fusion between synthetic membranes but do promote PEG-mediated membrane fusion. Biophys. J. 90 1661-1675 (2006).

23. Misura, K. M., Bock, J. B., Gonzalez, L. C. Jr, Scheller, R. H. \& Weis, W. I. Three-dimensional structure of the amino-terminal domain of syntaxin 6 , a SNAP-25 C homolog. Proc. Natl Acad. Sci. USA 99, 9184-9189 (2002).

24. Dietrich, L. E., Boeddinghaus, C., Lagrassa, T. J. \& Ungermann, C. Control of eukaryotic membrane fusion by N-terminal domains of SNARE proteins. Biochim. Biophys. Acta 1641, 111-119 (2003).

25. Gonzalez, L. C., Weis, W. I. \& Scheller, R. H. A novel SNARE N-terminal domain revealed by the crystal structure of Sec22b. J. Biol. Chem. 276, 24203-24211 (2001)

26. Tochio, H., Tsui, M. M., Banfield, D. K. \& Zhang, M. An autoinhibitory mechanism for nonsyntaxin SNARE proteins revealed by the structure of Ykt6p. Science 293, 698-702 (2001).

27. Lu, J., Garcia, J., Dulubova, I., Sudhof, T. C. \& Rizo, J. Solution structure of the Vam7p PX domain. Biochemistry 41, 5956-5962 (2002).

28. Calakos, N., Bennett, M. K., Peterson, K. E. \& Scheller, R. H. Protein-protein interactions contributing to the specificity of intracellular vesicular trafficking. Science 263, 1146-1149 (1994).

29. Dulubova, I. et al. A conformational switch in syntaxin during exocytosis: role of munc18. EMBO J. 18, 4372-4382 (1999).

30. Toonen, R. F. \& Verhage, M. Vesicle trafficking pleasure and pain from SM genes. Trends Cell Biol. 13, 177-186 (2003)

31. Dietrich, L. E., Gurezka, R., Veit, M. \& Ungermann, C. The SNARE Ykt6 mediates protein palmitoylation during an early stage of homotypic vacuole fusion. EMBO J. 23, 45-53 (2004).

32. Linder, M. E. $\&$ Deschenes, R. J. Model organisms lead the way to protein palmitoyltransferases. J. Cell Sci. 117, 521-526 (2004).

33. Munson, M., Chen, X., Cocina, A. E., Schultz, S. M. \& Hughson, F. M. Interactions within the yeast t-SNARE Sso $1 p$ that control SNARE complex assembly. Nature Struct. Biol. 7, 894-902 (2000).

34. Van Komen, J. S., Bai, X., Scott, B. L. \& McNew, J. A. An intramolecular t-SNARE complex functions in vivo without the syntaxin $\mathrm{NH}_{2}$-terminal regulatory domain. J. Cell Biol. 172, 295-307 (2006). Shows that the requirement for the $\mathrm{N}$-termina three-helix-bundle domain of a Qa-SNARE in $S$. cerevisiae exocytosis can be bypassed when $\mathrm{Q}$-(t)-SNARE motifs are fused together.

35. Wang, Y., Dulubova, I., Rizo, J. \& Südhof, T. C. Functional analysis of conserved structural elements in yeast syntaxin Vam3p. J. Biol. Chem. 276 28598-28605 (2001)

36. Südhof, T. C., De Camilli, P., Niemann, H. \& Jahn, R. Membrane fusion machinery: insights from synaptic proteins. Cell 75, 1-4 (1993).

37. Pelham, H. R., Banfield, D. K. \& Lewis, M. J. SNAREs involved in traffic through the Golgi complex. Cold Spring Harb. Symp. Quant. Biol. 60, 105-111 (1995).

38. Mayer, A., Wickner, W. \& Haas, A. Sec18p (NSF)driven release of $\operatorname{Sec} 17 p(\alpha-S N A P)$ can precede docking and fusion of yeast vacuoles. Cell 85, 83-94 (1996).

By studying the in vitro fusion of vacuoles, these authors show that NSF is not needed for fusion but functions as an activation or priming factor.

39. Hanson, P. I., Roth, R., Morisaki, H., Jahn, R. \& Heuser, J. E. Structure and conformational changes in NSF and its membrane receptor complexes visualized by quick-freeze/deep-etch electron microscopy. Cell 90, 523-535 (1997).

40. Lin, R. C. \& Scheller, R. H. Structural organization of the synaptic exocytosis core complex. Neuron 19 , 1087-1094 (1997).

References 39 and 40 show that neurona SNAREs are aligned in parallel in the SNARE complex, which led to the proposal of the 'zippering' hypothesis of SNARE function.

41. Pennuto, M., Bonanomi, D., Benfenati, F. \& Valtorta, F. Synaptophysin I controls the targeting of VAMP2/ synaptobrevin II to synaptic vesicles. Mol. Biol. Cell 14, 4909-4919 (2003).

42. Mossessova, E., Bickford, L. C. \& Goldberg, J. SNARE selectivity of the COPII coat. Cell 114, 483-495 (2003).
43. Peden, A. A., Park, G. Y \& Scheller, R. H. The di-leucine motif of vesicle-associated membrane protein 4 is required for its localization and AP- 1 binding. J. Biol. Chem. 276, 49183-49187 (2001).

44. Siniossoglou, S. \& Pelham, H. R. An effector of Ypt6p binds the SNARE TIg $1 p$ and mediates selective fusion of vesicles with late Golgi membranes. EMBO J. 20 , 5991-5998 (2001)

45. Collins, K. M., Thorngren, N. L., Fratti, R. A. \& Wickner, W. T. Sec17p and HOPS, in distinct SNARE complexes, mediate SNARE complex disruption or assembly for fusion. EMBO J. 24, 1775-1786 (2005).

46. Kweon, D. H., Kim, C. S. \& Shin, Y. K. The membrane dipped neuronal SNARE complex: a site-directed spin labeling electron paramagnetic resonance study. Biochemistry 41, 9264-9268 (2002).

47. Hu, K. et al. Vesicular restriction of synaptobrevin suggests a role for calcium in membrane fusion. Nature 415, 646-650 (2002)

48. Nevins, A. K. $\&$ Thurmond, D. C. A direct interaction between $\mathrm{Cdc} 42$ and vesicle-associated membrane protein 2 regulates SNARE-dependent insulin exocytosis. J. Biol. Chem. 28, 1944-1952 (2004).

49. Lang, T., Margittai, M., Holzler, H. \& Jahn, R. SNAREs in native plasma membranes are active and readily form core complexes with endogenous and exogenous SNAREs. J. Cell Biol. 158, 751-760 (2002).

50. Walch-Solimena, C. et al. The t-SNAREs syntaxin 1 and SNAP-25 are present on organelles that participate in synaptic vesicle recycling. J. Cell Biol. 128, 637-645 (1995).

51. Lang, T. et al. SNAREs are concentrated in cholesterol-dependent clusters that define docking and fusion sites for exocytosis. $E M B O \mathrm{~J} . \mathbf{2 0}$ 2202-2213 (2001)

52. Chamberlain, L. H., Burgoyne, R. D. \& Gould, G. W. SNARE proteins are highly enriched in lipid rafts in PC12 cells: implications for the spatial control of exocytosis. Proc. Natl Acad. Sci. USA 98, 5619-5624 (2001)

53. Predescu, S. A., Predescu, D. N., Shimizu, K., Klein, I. K. \& Malik, A. B. Cholesterol-dependent syntaxin- 4 and SNAP-23 clustering regulates caveolae fusion with the endothelial plasma membrane. J. Biol. Chem. 280, 37130-37138 (2005).

54. Laage, R., Rohde, J., Brosig, B. \& Langosch, D. A conserved membrane-spanning amino acid motif drives homomeric and supports heteromeric assembly of presynaptic SNARE proteins. J. Biol. Chem. 275, 17481-17487 (2000)

55. Fiebig, K. M., Rice, L. M., Pollock, E. \& Brunger, A. T. Folding intermediates of SNARE complex assembly. Nature Struct. Biol. 6, 117-123 (1999).

56. Fasshauer, D. \& Margittai, M. A transient N-terminal interaction of SNAP-25 and syntaxin nucleates SNARE assembly. J. Biol. Chem. 279, 7613-7621 (2004).

57. Nicholson, K. L. et al. Regulation of SNARE complex assembly by an $\mathrm{N}$-terminal domain of the t-SNARE Sso 1 p. Nature Struct. Biol. 5, 793-802 (1998).

58. Peng, R. \& Gallwitz, D. Sly 1 protein bound to Golgi syntaxin Sed5p allows assembly and contributes to specificity of SNARE fusion complexes. J. Cell Biol. 157, 645-655 (2002).

59. Bryant, N. J. \& James, D. E. Vps45p stabilizes the syntaxin homologue TIg2p and positively regulates SNARE complex formation. EMBO J. 20 , 3380-3388 (2001).

60. Misura, K. M., May, A. P. \& Weis, W. I. Proteinprotein interactions in intracellular membrane fusion. Curr. Opin. Struct. Biol. 10, 662-671 (2000).

61. Weimer, R. M. et al. Defects in synaptic vesicle docking in unc- 18 mutants. Nature Neurosci. 6 , 1023-1030 (2003).

62. Voets, T. et al. Munc18-1 promotes large dense-core vesicle docking. Neuron 31, 581-591 (2001).

63. Grote, E., Carr, C. M. \& Novick, P. J. Ordering the final events in yeast exocytosis. J. Cell Biol. 151, 439-452 (2000)

64. An, S. J. \& Almers, W. Tracking SNARE complex formation in live endocrine cells. Science 306, 1042-1046 (2004)

65. Chen, Y. A., Scales, S. J. \& Scheller, R. H. Sequentia SNARE assembly underlies priming and triggering of exocytosis. Neuron 30, 161-170 (2001).

66. Kraynack, B. A. et al. Dsl1p, Tip20p, and the novel DsI3(Sec39) protein are required for the stability of the Q/t-SNARE complex at the endoplasmic reticulum in yeast. Mol. Biol. Cell 16, 3963-3977 (2005).

67. Hatsuzawa, K., Lang, T., Fasshauer, D., Bruns, D. \& Jahn, R. The R-SNARE motif of tomosyn forms SNARE core complexes with syntaxin 1 and SNAP-25 and down-regulates exocytosis. J. Biol. Chem. 278, 31159-31166 (2003)

68. Sakisaka, T. et al. Regulation of SNAREs by tomosyn and ROCK: implication in extension and retraction of neurites. J. Cell Biol. 166, 17-25 (2004).

69. Pobbati, A. V., Razeto, A., Boddener, M., Becker, S. \& Fasshauer, D. Structural basis for the inhibitory role of tomosyn in exocytosis. J. Biol. Chem. 279, 47192-47200 (2004).

70. Scales, S. J., Hesser, B. A., Masuda, E. S. \& Scheller, R. H. Amisyn, a novel syntaxin-binding protein that may regulate SNARE complex assembly. J. Biol. Chem. 277, 28271-28279 (2002).

71. Echarri, A., Lai, M. J., Robinson, M. R. \& Pendergast, A. M. Abl interactor 1 (Abi-1) wave-binding and SNARE domains regulate its nucleocytoplasmic shuttling, lamellipodium localization, and wave-1 levels. Mol. Cell. Biol. 24 4979-4993 (2004)

72. Nichols, B. J., Ungermann, C., Pelham, H. R. Wickner, W. T. \& Haas, A. Homotypic vacuolar fusion mediated by t- and v-SNAREs. Nature 387 199-202 (1997).

By studying the in vitro fusion of vacuoles as a model, this work shows that fusion requires an interaction between complementary sets of SNAREs in the fusing membranes.

73. Chen, Y. A., Scales, S. J., Patel, S. M., Doung, Y. C. \& Scheller, R. H. SNARE complex formation is triggered by $\mathrm{Ca}^{2+}$ and drives membrane fusion. Cell $\mathbf{9 7}$, 165-174 (1999)

74. Sørensen, J. B. Formation, stabilisation and fusion of the readily releasable pool of secretory vesicles. Pflugers Arch. 448, 347-362 (2004).

75. Borisovska, M. et al. v-SNAREs control exocytosis of vesicles from priming to fusion. $E M B O \mathrm{~J} . \mathbf{2 4}$ 2114-2126 (2005)

76. Chapman, E. R. Synaptotagmin: $\mathrm{a} \mathrm{Ca}^{2+}$ sensor that triggers exocytosis? Nature Rev. Mol. Cell Biol. 3 498-508 (2002)

77. Südhof, T. C. The synaptic vesicle cycle. Annu. Rev. Neurosci. 27, 509-547 (2004)

78. Südhof, T. C. Synaptotagmins: why so many? J. Biol. Chem. 277, 7629-7632 (2002).

79. Reim, K. et al. Complexins regulate a late step in $\mathrm{Ca}^{2+}$-dependent neurotransmitter release. Cell 104 71-81 (2001).

80. Chen, X. et al. Three-dimensional structure of the complexin/SNARE complex. Neuron 33, 397-409 (2002).

81 Jahn, R. \& Grubmuller, H. Membrane fusion. Curr. Opin. Cell Biol. 14, 488-495 (2002).

82. Chernomordik, L. V. \& Kozlov, M. M. Protein-lipid interplay in fusion and fission of biological membranes. Annu. Rev. Biochem. 72, 175-207 (2003).

83. Kiessling, V. \& Tamm, L. K. Measuring distances in supported bilayers by fluorescence interference contrast microscopy: polymer supports and SNARE proteins. Biophys. J. 84, 408-418 (2003).

84. Knecht, V. \& Grubmüller, H. Mechanical coupling via the membrane fusion SNARE protein syntaxin $1 \mathrm{~A}$ a molecular dynamics study. Biophys. J. 84 , 1527-1547 (2003)

85. Grote, E., Baba, M., Ohsumi, Y. \& Novick, P. J. Geranylgeranylated SNAREs are dominant inhibitors of membrane fusion. J. Cell Biol. 151, 453-466 (2000).

86. McNew, J. A., Weber, T., Engelman, D. M. Söllner, T. H. \& Rothman, J. E. The length of the flexible SNAREpin juxtamembrane region is a critica determinant of SNARE-dependent fusion. Mol. Cell 4 415-421 (1999)

87. Montecucco, C., Schiavo, G. \& Pantano, S. SNARE complexes and neuroexocytosis: how many, how close? Trends Biochem. Sci. 30, 367-372 (2005)

88. Xu, Y., Zhang, F., Su, Z., McNew, J. A. \& Shin, Y. K. Hemifusion in SNARE-mediated membrane fusion. Nature Struct. Mol. Biol. 12, 417-422 (2005).

89. Reese, C., Heise, F. \& Mayer, A. Trans-SNARE pairing can precede a hemifusion intermediate in intracellular membrane fusion. Nature $\mathbf{4 3 6}$, 410-414 (2005)

90. Giraudo, C. G. et al. SNAREs can promote complete fusion and hemifusion as alternative outcomes. J. Cell Biol. 170, 249-260 (2005). 
91. Bentz, J. Membrane fusion mediated by coiled coils: a hypothesis. Biophys. J. 78, 886-900 (2000)

92. Langosch, D. et al. Peptide mimics of SNARE transmembrane segments drive membrane fusion depending on their conformational plasticity. J. Mol. Biol. 311, 709-721 (2001).

93. Nelson, N. A journey from mammals to yeast with vacuolar $\mathrm{H}^{+}$-ATPase (V-ATPase). J. Bioenerg. Biomembr. 35, 281-289 (2003)

94. Mayer, A. Membrane fusion in eukaryotic cells. Annu. Rev. Cell Dev. Biol. 18, 289-314 (2002).

95. Hiesinger, P. R. et al. The v-ATPase $\mathrm{V}_{0}$ subunit a1 is required for a late step in synaptic vesicle exocytosis in Drosophila. Cell 121, 607-620 (2005).

96. Peters, C. et al. Trans-complex formation by proteolipid channels in the terminal phase of membrane fusion. Nature 409, 581-588 (2001).

97. Bayer, M. J., Reese, C., Buhler, S., Peters, C. \& Mayer, A. Vacuole membrane fusion: $V_{0}$ functions after trans-SNARE pairing and is coupled to the $\mathrm{Ca}^{2+}$ releasing channel. J. Cell Biol. 162, 211-222 (2003).

98. Hanson, P. I. \& Whiteheart, S. W. AAA + proteins: have engine, will work. Nature Rev. Mol. Cell Biol. 6 , 519-529 (2005).

99. Fasshauer, D., Antonin, W., Subramaniam, V. \& Jahn, R. SNARE assembly and disassembly exhibit a pronounced hysteresis. Nature Struct. Biol. 9 144-151 (2002)

100. Marz, K. E., Lauer, J. M. \& Hanson, P. I. Defining the SNARE complex binding surface of $\alpha$-SNAP: implications for SNARE complex disassembly. J. Biol. Chem. 278, 27000-27008 (2003)

101. Scales, S. J., Yoo, B. Y. \& Scheller, R. H. The ionic layer is required for efficient dissociation of the SNARE complex by $\alpha$-SNAP and NSF. Proc. NatI Acad. Sci. USA 98, 14262-14267 (2001).

102. Martin, A., Baker, T. A. \& Sauer, R. T. Rebuilt AAA + motors reveal operating principles for ATP-fuelled machines. Nature 437, 1115-1120 (2005).

103. Hanson, P. I., Otto, H., Barton, N. \& Jahn, R. The $N$-ethylmaleimide-sensitive fusion protein and $\alpha$-SNAP induce a conformational change in syntaxin J. Biol. Chem. 270, 16955-16961 (1995).

104. McMahon, H. T. \& Südhof, T. C. Synaptic core complex of synaptobrevin, syntaxin, and SNAP25 forms high affinity $\alpha$-SNAP binding site. J. Biol. Chem. 270, 2213-2217 (1995).

105. Wickner, W. \& Haas, A. Yeast homotypic vacuole fusion: a window on organelle trafficking mechanisms. Annu. Rev. Biochem. 69, 247-275 (2000).

106. Ungermann, C. \& Langosch, D. Functions of SNAREs in intracellular membrane fusion and lipid bilaye mixing. J. Cell Sci. 118, 3819-3828 (2005).

107. Sogaard, M. et al. A rab protein is required for the assembly of SNARE complexes in the docking of transport vesicles. Cell 78, 937-948 (1994).

108. Littleton, J. T. et al. Temperature-sensitive paralytic mutations demonstrate that synaptic exocytosis requires SNARE complex assembly and disassembly. Neuron 21, 401-413 (1998)

109. Hay, J. C. et al. Localization, dynamics, and protein interactions reveal distinct roles for ER and Golgi SNAREs. J. Cell Biol. 141, 1489-1502 (1998).

110. Cao, X. \& Barlowe, C. Asymmetric requirements for a Rab GTPase and SNARE proteins in fusion of COPII vesicles with acceptor membranes. J. Cell Biol. 149, 55-66 (2000)

111. Antonin, W., Holroyd, C., Tikkanen, R., Honing, S. \& Jahn, R. The R-SNARE endobrevin/VAMP-8 mediates homotypic fusion of early endosomes and late endosomes. Mol. Biol. Cell 11, 3289-3298 (2000).

112. West, A. E., Neve, R. L. \& Buckley, K. M. Targeting of the synaptic vesicle protein synaptobrevin in the axon of cultured hippocampal neurons: evidence for two distinct sorting steps. J. Cell Biol. 139, 917-927 (1997).

113. Zeng, Q., Tran, T. T., Tan, H. X. \& Hong, W The cytoplasmic domain of Vamp 4 and Vamp5 is responsible for their correct subcellular targeting: the $\mathrm{N}$-terminal extension of Vamp4 contains a dominant autonomous targeting signal for the trans-Golgi network. J. Biol. Chem. 278, 23046-23054 (2003).

114. Grote, E., Hao, J. C., Bennett, M. K. \& Kelly, R. B. A targeting signal in VAMP regulating transport to synaptic vesicles. Cell 81, 581-589 (1995).

115. Pfeffer, S. \& Aivazian, D. Targeting Rab GTPases to distinct membrane compartments. Nature Rev. Mol. Cell Biol. 5, 886-896 (2004).

116. Fasshauer, D., Antonin, W., Margittai, M., Pabst, S. \& Jahn, R. Mixed and non-cognate SNARE complexes.
Characterization of assembly and biophysical properties. J. Biol. Chem. 274, 15440-15446 (1999).

117. Yang, B. et al. SNARE interactions are not selective. Implications for membrane fusion specificity. J. Biol. Chem. 274, 5649-5653 (1999).

118. Parlati, F. et al. Topological restriction of SNAREdependent membrane fusion. Nature 407, 194-198 (2000).

119. Paumet, F., Rahimian, V. \& Rothman, J. E. The specificity of SNARE-dependent fusion is encoded in the SNARE motif. Proc. Natl Acad. Sci. USA 101, 3376-3380 (2004).

120. Brandhorst, D. et al. Homotypic fusion of early endosomes: SNAREs do not determine fusion specificity. Proc. Natl Acad. Sci. USA 103. 2701-2706 (2006).

121. Liu, Y. \& Barlowe, C. Analysis of Sec22p in endoplasmic reticulum/Golgi transport reveals cellular redundancy in SNARE protein function. $\mathrm{Mol}$. Biol. Cell 13, 3314-3324 (2002).

122. Sørensen, J. B. et al. Differential control of the releasable vesicle pools by SNAP- 25 splice variants and SNAP-23. Cell 114, 75-86 (2003).

123. Fischer von Mollard, G. \& Stevens, T. H. The Saccharomyces cerevisiae $\mathrm{v}$-SNARE Vti $1 \mathrm{p}$ is required for multiple membrane transport pathways to the vacuole. Mol. Biol. Cell 10, 1719-1732 (1999).

124. Tsui, M. M. \& Banfield, D. K. Yeast Golgi SNARE interactions are promiscuous. J. Cell Sci. 113, 145-152 (2000)

125. Antonin, W. et al. A SNARE complex mediating fusion of late endosomes defines conserved properties of SNARE structure and function. EMBO J. 19 6453-6464 (2000).

126. Wang, C. C. et al. A role of VAMP8/endobrevin in regulated exocytosis of pancreatic acinar cells. Dev. Cell 7, 359-371 (2004)

127. Scales, S. J. et al. SNAREs contribute to the specificity of membrane fusion. Neuron 26 457-464 (2000)

128. Link, E. et al. Cleavage of cellubrevin by tetanus toxin does not affect fusion of early endosomes. J. Biol. Chem. 268, 18423-18426 (1993).

129. Weber, T. et al. SNAREpins: minimal machinery for membrane fusion. Cell 92, 759-772 (1998). Shows that SNAREs that are reconstituted into proteoliposomes are capable of fusing membranes without the need for other factors.

130. Schuette, C. G. et al. Determinants of liposome fusion mediated by synaptic SNARE proteins. Proc. Nat Acad. Sci. USA 101, 2858-2863 (2004).

131. Tucker, W. C., Weber, T. \& Chapman, E. R. Reconstitution of $\mathrm{Ca}^{2+}$-regulated membrane fusion by synaptotagmin and SNAREs. Science 304 435-438 (2004).

132. Mahal, L. K. Sequeira, S. M., Gureasko, J. M. \& Söllner, T. H. Calcium-independent stimulation of membrane fusion and SNAREpin formation by synaptotagmin I. J. Cell Biol. 158, 273-282 (2002).

133. Chen, X. et al. SNARE-mediated lipid mixing depends on the physical state of the vesicles. Biophys. J. 90, 2062-2074 (2005).

134. Fix, M. et al. Imaging single membrane fusion events mediated by SNARE proteins. Proc. Natl Acad. Sci. USA 101, 7311-7316 (2004)

135. Bowen, M. E., Weninger, K., Brunger, A. T. \& Chu, S Single molecule observation of liposome-bilayer fusion thermally induced by soluble $N$-ethyl maleimide sensitive-factor attachment protein receptors (SNAREs). Biophys. J. 87, 3569-3584 (2004).

136. Liu, T., Tucker, W. C., Bhalla, A., Chapman, E. R. \& Weisshaar, J. C. SNARE-driven, 25-millisecond vesicle fusion in vitro. Biophys. J. 89, 2473-2480 (2005).

137. Lerman, J. C., Robblee, J., Fairman, R. \& Hughson, F. M. Structural analysis of the neuronal SNARE protein syntaxin-1A. Biochemistry 39 8470-8479 (2000).

138. Misura, K. M., Scheller, R. H. \& Weis, W. I. Threedimensional structure of the neuronal-Sec 1 syntaxin 1a complex. Nature 404, 355-362 (2000).

139. Novick, P., Field, C. \& Schekman, R. Identification of 23 complementation groups required for posttranslational events in the yeast secretory pathway. Cell 21, 205-215 (1980)

Classical study in which many of the genes that are essential for various steps in the secretory pathway were identified for the first time.
140. Eakle, K. A. Bernstein, M. \& Emr S. D. Characterization of a component of the yeast secretion machinery: identification of the SEC18 gene product. Mol. Cell. Biol. 8, 4098-4109 (1988).

141. Wilson, D. W. et al. A fusion protein required for vesicle-mediated transport in both mammalian cells and yeast. Nature 339, 355-359 (1989).

142. Clary, D. O., Griff, I. C. \& Rothman, J. E. SNAPs, a family of NSF attachment proteins involved in intracellular membrane fusion in animals and yeast. Cell 61, 709-721 (1990).

References 141 and 142 describe the first characterization of NSF and SNAPs, respectively, as soluble factors that are required for intracellular membrane-fusion reactions.

143. Trimble, W. S., Cowan, D. M. \& Scheller, R. H. VAMP-1: a synaptic vesicle-associated integral membrane protein. Proc. Natl Acad. Sci. USA 85 4538-4542 (1988)

144. Baumert, M., Maycox, P. R., Navone, F., De Camilli, P. \& Jahn, R. Synaptobrevin: an integral membrane protein of 18,000 daltons present in small synaptic vesicles of rat brain. EMBO J. 8, 379-384 (1989).

45. Bennett, M. K., Calakos, N. \& Scheller, R. H Syntaxin: a synaptic protein implicated in docking of synaptic vesicles at presynaptic active zones. Science 257, 255-259 (1992).

146. Dascher, C., Ossig, R., Gallwitz, D. \& Schmitt, H. D. Identification and structure of four yeast genes (SLY) that are able to suppress the functional loss of YPT1 a member of the RAS superfamily. Mol. Cell. Biol. 11, 872-885 (1991).

147. Gerst, J. E., Rodgers, L., Riggs, M. \& Wigler, M. SNC1, a yeast homolog of the synaptic vesicleassociated membrane protein/synaptobrevin gene family: genetic interactions with the $R A S$ and $C A P$ genes. Proc. Natl Acad. Sci. USA 89, 4338-4342 (1992).

148. Bennett, M. K. \& Scheller, R. H. The molecular machinery for secretion is conserved from yeast to neurons. Proc. Natl Acad. Sci. USA 90, 2559-2563 (1993)

149. Schiavo, G. et al. Tetanus and botulinum-B neurotoxins block neurotransmitter release by proteolytic cleavage of synaptobrevin. Nature 359 832-835 (1992)

VAMP/synaptobrevin is shown to be proteolysed by the tetanus and botulinum neurotoxins. Together with references 150 and 151, this work shows that the toxins block membrane fusion by cleaving neuronal SNAREs.

150. Blasi, J. et al. Botulinum neurotoxin A selectively cleaves the synaptic protein SNAP-25. Nature 365 160-163 (1993)

151. Blasi, J. et al. Botulinum neurotoxin C1 blocks neurotransmitter release by means of cleaving HPC1/syntaxin. EMBO J. 12, 4821-4828 (1993).

152. Söllner, T. et al. SNAP receptors implicated in vesicle targeting and fusion. Nature 362, 318-324 (1993). The first identification of a complex of VAMP/synaptobrevin, SNAP-25 and syntaxin as a receptor for NSF and $\alpha$-SNAP, and the introduction of the term SNARE.

\section{Acknowledgements}

The authors are indebted to D. Fasshauer, E. Neher, S Rizzoli and J. Sorensen for helpful comments and for critically reading the manuscript. We hope our colleagues will understand that, due to space limitations, only a fraction of the relevant work could be discussed. R.J. is supported by an award from the Gottfried Wilhelm Leibniz Program of the Deutsche Forschungsgemeinschaft and by a grant from the National Institutes of Health.

Competing interests statement

The authors declare no competing financial interests.

\section{DATABASES}

The following terms in this article are linked online to: UniProtKB: http://ca.expasy.org/sprot NSF | Sec22 | SNAP-25 | synaptotagmin | Tlg1 | Tlg2 | Vac8 | Vam3|Vam7|Ykt

FURTHER INFORMATION

Reinhard Jahn's homepage:

http://www.mpg.de/cgi-bin/mpg.de/person.cgi?persld= 163058\&lang=de\&inst=biophysikalische_chemie Richard H. Scheller's homepage: http://www.gene.com/ gene/about/management/exec/scheller.jsp Access to this links box is available online. 